\title{
Mesothelin and osteopontin
}

\author{
To the Editor:
}

The review by Pantazopoulos et al. [1] on mesothelin and osteopontin was timely and well written. However, I would like to comment on some of the statements that were made. Firstly, it states that "patients with early-stage disease can survive for more than 5 years if the tumour is promptly resected". This implies that resection would benefit the patient, which is far from proven; there is no randomised study showing this, except for the much criticised the Mesothelioma and Radical Surgery (MARS) study [2] in England, which showed that resection considerably shortened survival. The good survival seen by SUGARBAKER et al. [3] and in other studies can be explained by very strict selection and these patients are likely to survive at least as long without resection, as seen from studies where "operable" patients were treated conservatively. Furthermore, the authors claim that "early diagnosis offers the best hope for a favourable prognosis", which is an interesting but unproven hypothesis. There is of course a lead time bias, the earlier the diagnosis the longer the survival, but unfortunately we do not know whether early intervention will in fact prolong survival. Thus, screening for mesothelioma in risk groups is not indicated at present, screening should only be performed in diseases where the prognosis has been shown to be improved by early discovery.

I fully agree with the other main conclusions in the review, namely that mesothelin, in serum or pleura, can be a useful aid in the diagnostic setup and also used for monitoring patients for recurrence.

O

@ERSpublications

Early stage mesothelioma and surgery: where do we stand? http://ow.ly/kRTJ2

Gunnar Hillerdal

Dept of Pulmonary Medicine, Karolinska Hospital, Stockholm, Sweden.

Correspondence: G. Hillerdal, Karolinska Hospital, 17176 Stockholm, Stockholm, Sweden.

E-mail: gunnar.hillerdal@karolinska.se.

Received: March 232013 | Accepted: April 32013

Conflict of interest: None declared.

\section{References}

1 Pantazopoulos I, Boura P, Xanthos T, et al. Effectiveness of mesothelin family proteins and osteopontin for malignant mesothelioma. Eur Respir J 2013; 41: 706-715.

2 Treasure T, Lang-Lazdunski L, Waller D, et al. Extra-pleural pneumonectomy versus no extra-pleural pneumonectomy for patients with malignant pleural mesothelioma: clinical outcomes of the Mesothelioma and Radical Surgery (MARS) randomised feasibility study. Lancet Oncol 2011; 12: 763-772.

3 Sugarbaker DJ, Flores RM, Jaklitsch MT, et al. Resection margins, extrapleural nodal status, and cell type determine postoperative long-term survival in trimodality therapy of malignant pleural mesothelioma: results in 183 patients. J Thorac Cardiovasc Surg 1999; 117: 54-63.

Eur Respir J 2013; 42: 557 | DOI: 10.1183/09031936.00052213 | Copyright @ERS 2013

From the authors:

We would like to thank G. Hillerdal for his interest in our article [1] and for giving us the opportunity to make some comments regarding multimodality therapy and early stage disease.

SUGARBAKER et al. [2] reported a groundbreaking result in 1999: patients with early stage malignant pleural mesothelioma (MPM) had a 5-year survival rate after trimodality therapy that exceeded $40 \%$. Since then, there have been a number of subsequent prospective and retrospective series, which have all demonstrated a median survival of 16.8-25.5 months [3-8]. Moreover, on September 11, 2012, in Boston (MA, USA) when the International Mesothelioma Interest Group met to discuss the role of surgery in the treatment of MPM, Valerie Rusch (New York, NY, USA) presented a preliminary analysis of the International Association for the Study of Lung Cancer (IASLC) staging project $[9,10]$. In the IASLC worldwide registry of patients with all stages of epithelial MPM, the analysis showed a 19-month median survival among 1359 patients 
undergoing surgical resection. Moreover, patients undergoing extrapleural pneumonectomy for early-stage disease demonstrated survival superior to that of all other subgroups, a median of 40 months [9].

This finding, that early disease may be effectively treated, emphasises the importance of identifying a tumour marker that is practical for screening and can allow physicians to make an early diagnosis. In our review we clearly state that mesothelin family proteins and osteopontin have limited sensitivity for identifying early stage disease, and this might limit their use in screening. Furthermore, in the near future, their application in clinical practice is most probably in monitoring response to therapy, rather than in risk assessment for asbestos-exposed populations.

We fully agree that the Mesothelioma and Radical Surgery (MARS) I trial, which concluded that extrapleural pneumonectomy offers no benefit, was characterised by strong biases which make drawing any conclusion impossible [11]. On the basis of the current literature and the IASLC report we believe that "there is life after MARS". International Mesothelioma Interest Group members also concluded that surgery should be performed in the multimodality treatment of MPM [9].

Mesothelioma is a heterogeneous disease and some long-term survivors clearly benefit from surgery. Future studies must explore new markers that will predict for resection only those patients most likely to achieve long-term survival. Well-designed randomised studies are needed, accompanied by a careful interpretation of the results, to avoid movement of clinical research for mesothelioma in the wrong direction.

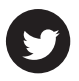
@ERSpublications

Early stage mesothelioma and surgery: where do we stand? http://ow.ly/kTms5

Ioannis Pantazopoulos ${ }^{1}$, Theodoros Xanthos ${ }^{2}$, Paraskevi Boura ${ }^{3}$ and Konstantinos Syrigos ${ }^{3}$

${ }^{1} 12$ th Dept of Respiratory Medicine, Sotiria General Hospital, Athens, ${ }^{2} \mathrm{MSc}$ Programme "Cardiopulmonary Resuscitation", University of Athens, Medical School, Athens, and ${ }^{3}$ Oncology Unit, 3rd Dept of Medicine, School of Medicine, University of Athens, Sotiria General Hospital, Athens, Greece.

Correspondence: I. Pantazopoulos, 12th Dept of Respiratory Medicine, Sotiria General Hospital, 152 Mesogeion Ave, 11527, Athens, Greece. E-mail: pantazopoulosioannis@yahoo.com

Received: April 112013 | Accepted: April 152013

Conflict of interest: None declared.

\section{References}

1 Pantazopoulos I, Boura P, Xanthos T, et al. Effectiveness of mesothelin family proteins and osteopontin for malignant mesothelioma. Eur Respir J 2013; 41: 706-715.

2 Sugarbaker DJ, Flores RM, Jaklitsch MT, et al. Resection margins, extrapleural nodal status, and cell type determine postoperative long-term survival in trimodality therapy of malignant pleural mesothelioma: results in 183 patients. J Thorac Cardiovasc Surg 1999; 117: 54-63.

3 Weder W, Stahel RA, Bernhard J, et al. Multicenter trial of neo-adjuvant chemotherapy followed by extrapleural pneumonectomy in malignant pleural mesothelioma. Ann Oncol 2007; 18: 1196-1202.

4 Weder W, Kestenholz P, Taverna C, et al. Neoadjuvant chemotherapy followed by extrapleural pneumonectomy in malignant pleural mesothelioma. J Clin Oncol 2004; 22: 3451-3457.

5 Rea F, Marulli G, Bortolotti L, et al. Induction chemotherapy, extrapleural pneumonectomy (EPP) and adjuvant hemi-thoracic radiation in malignant pleural mesothelioma (MPM): feasibility and results. Lung Cancer 2007; 57: 89-95.

6 Batirel HF, Metintas M, Caglar HB, et al. Trimodality treatment of malignant pleural mesothelioma. J Thorac Oncol 2008; 3: 499-504.

$7 \quad$ Krug LM, Pass HI, Rusch VW, et al. Multicenter phase II trial of neoadjuvant pemetrexed plus cisplatin followed by extrapleural pneumonectomy and radiation for malignant pleural mesothelioma. J Clin Oncol 2009; 27: 3007-3013.

8 Van Schil PE, Baas P, Gaafar R, et al. Trimodality therapy for malignant pleural mesothelioma: results from an EORTC phase II multicentre trial. Eur Respir J 2010; 36: 1362-1369.

9 Rusch V, Baldini EH, Bueno R, et al. The role of surgical cytoreduction in the treatment of malignant pleural mesothelioma: meeting summary of the International Mesothelioma Interest Group Congress, September 11-14, 2012, Boston, Mass. J Thorac Cardiovasc Surg 2013; 145: 909-910.

10 Rusch VW, Giroux D, Kennedy C, et al. Initial analysis of the international association for the study of lung cancer mesothelioma database. J Thorac Oncol 2012; 7: 1631-1639.

11 Treasure T, Lang-Lazdunski L, Waller D, et al. Extra-pleural pneumonectomy versus no extra-pleural pneumonectomy for patients with malignant pleural mesothelioma: clinical outcomes of the Mesothelioma and Radical Surgery (MARS) randomised feasibility study. Lancet Oncol 2011; 12: 763-772. 